\title{
Dynamique de l'occupation des terres et état de la flore et de la végétation dans le bassin supérieur de l'Alibori au Benin
}

\author{
AROUNA Ousséni183, ETENE Cyr Gervais² \& ISSIAKO Dramane ${ }^{3}$ \\ 1: Ecole des Sciences et Techniques du Bâtiment et de la Route (ESTBR), Université Nationale des Sciences, \\ Technologies, Ingénierie et Mathématiques (UNSTIM), 03 BP 304 Abomey, \\ E-mail : arounaousseni@gmail.com \\ 2 : Laboratoire Pierre Pagney "Climat, Eau, Ecosystèmes et Développement" (LACEEDE), Université d'Abomey-Calavi, \\ BP 922, Abomey-Calavi, Bénin \\ 3 : Laboratoire de Cartographie (LaCarto), Université d'Abomey-Calavi, 10 BP 1082, Cotonou, Bénin.
}

Original submitted in on $18^{\text {th }}$ November 2016. Published online at www. m.elewa.org on $31^{\text {st }}$ December 2016 http://dx.doi.org/10.4314/jab.v108i1.7

\section{RESUME}

La flore et la végétation constituent des indicateurs importants de l'état de santé d'un bassin hydrographique. L'objectif global de la présente recherche est d'évaluer les effets des changements spatio-temporels de l'occupation des terres sur la flore et la végétation du bassin supérieur de l'Alibori. La cartographie diachronique de l'occupation des terres et les inventaires phytosociologique et forestier sont les principales méthodes utilisées. Les résultats obtenus ont révélé une réduction de près de $40 \%$ de la superficie des formations végétales naturelles (forêts claires, savanes boisées, savanes arborées et savanes arbustives) au profit de celle des formations anthropiques (mosaïques de champs et jachères et agglomérations) entre 1985 et 2016. Les formations naturelles résiduelles sont établies sur des terres marginales inaptes à l'agriculture. La caractérisation de la flore et de la végétation a permis de recenser 183 espèces réparties en 129 genres et 53 familles. Les espèces soudaniennes sont les plus abondantes et les plus dominantes suivies de très près par les espèces pantropicales. L'indice de diversité de Shannon varie de 1,5 à 3 bits. La richesse spécifique varie de 9 à 30 espèces par placeau. Ces valeurs relativement faibles de la richesse spécifique et de l'indice de diversité de Shannon confirment bien l'état de dégradation de la végétation déjà révélées par les images satellites dans ce bassin versant.

Mots clés : végétation, cartographie, dégradation, Alibori, Bénin.

\begin{abstract}
Land cover dynamics and state of flora and vegetation in the upper basin of Alibori in Benin

Flora and vegetation are important indicators of the health state of a watershed. The aim of this research is to assess the effects of time-space changes of land cover on the flora and vegetation in the Upper Alibori Basin. Land cover diachronic mapping and phytosociological and forest inventories are the main methods used. The results obtained revealed a reduction of $40 \%$ of natural vegetation (woodlands, savannas woodlands, tree savannas and shrub savannas) in favor of mosaics of fields and fallows and settlements between 1985 and 2016. Residual natural formations are established on marginal lands unrelevent to agriculture. The
\end{abstract}


characterization of flora and vegetation made it possible to identify 183 species divided in 129 genera and 53 families. Sudanian species are the most abundant and dominant, followed closely by pantropical species. The Shannon Diversity Index varies from 1.5 to 3 bits. Species richness varies from 9 to 30 species per plot. These relatively low values of specific richness and Shannon's diversity index confirm this state of vegetation degradation revealed by satellite images in this watershed.

Keywords : vegetation, mapping, degradation, Alibori, Benin.

\section{INTRODUCTION}

Dans le contexte actuel des changements globaux, les modifications spatio-temporelles de l'occupation des terres surtout celles de la végétation sont devenues des indicateurs qui permettent d'évaluer la santé des écosystèmes. Ainsi, la végétation est certainement devenue la composante des surfaces terrestres la plus intensivement surveillée par satellite (Arouna, 2012). Selon Sow (2012), l'état de la flore et de la végétation est extrêmement dynamique et sensible aux changements notamment les modifications de l'occupation des terres. Le bassin versant supérieur de l'Alibori connaît de plus en plus des changements spatio-temporels de l'occupation des terres (Mama, 2013 ; Issiako, 2015). Dans ce bassin versant se trouvent à la fois les terroirs villageois destinés aux activités agropastorales et la forêt classée de l'Alibori Supérieur dédiée à la conservation de la biodiversité. Une portion du bassin versant supérieur de la rivière Alibori a été érigée en aire protégée notamment en forêt classée par l'arrêté $N^{\circ} 6459$ SE du 18 août 1955. Les objectifs assignés à cette forêt classée est la protection du bassin versant de la rivière Alibori

\section{MATERIEL ET METHODES}

Présentation du milieu d'étude : La figure 1 présente le milieu d'étude constitué de la portion du bassin supérieur de la rivière Alibori se trouvant dans les communes de Banikoara et de Gogounou. II est localisé entre $10^{\circ} 41$ et $11^{\circ} 11$ de latitude Nord et entre $2^{\circ} 15$ et $2^{\circ} 48$ de longitude
(Toko Imorou et al., 2010). Mais cette forêt classée est ceinturée par les Communes de Banikoara, de Gogounou, de Kandi et de Kèrou constituant le bassin cotonnier du Bénin. La forêt classée de l'Alibori Supérieur et les terroirs villageois sont majoritairement occupés par les activités agropastorales et d'exploitation forestière (Issiako, 2015). Ces derniers connaissent également une surexploitation des terres agricoles et des ressources forestières (Mama et al., 2013; Sabi Monra, 2015). Dans un tel contexte de fortes pressions anthropiques, il est alors opportun d'évaluer les effets de l'occupation des terres sur la flore et la végétation. L'objectif de la présente recherche est d'évaluer les effets de la dynamique de l'occupation des terres sur la biodiversité végétale. L'hypothèse de recherche stipule que la dynamique régressive de l'occupation des terres induit une baisse de la diversité spécifique et une perturbation de la répartition des espèces végétales. Le matériel et les méthodes, les résultats et la discussion constituent les principales sections du présent article.

Est et couvre une superficie de $2026,22 \mathrm{Km}^{2}$. Le bassin versant supérieur de la rivière Alibori abrite une partie importante de la forêt classée de l'Alibori supérieur (56458 ha) et des terroirs villageois (terroirs des Arrondissements de Bagou et de Goumori). 


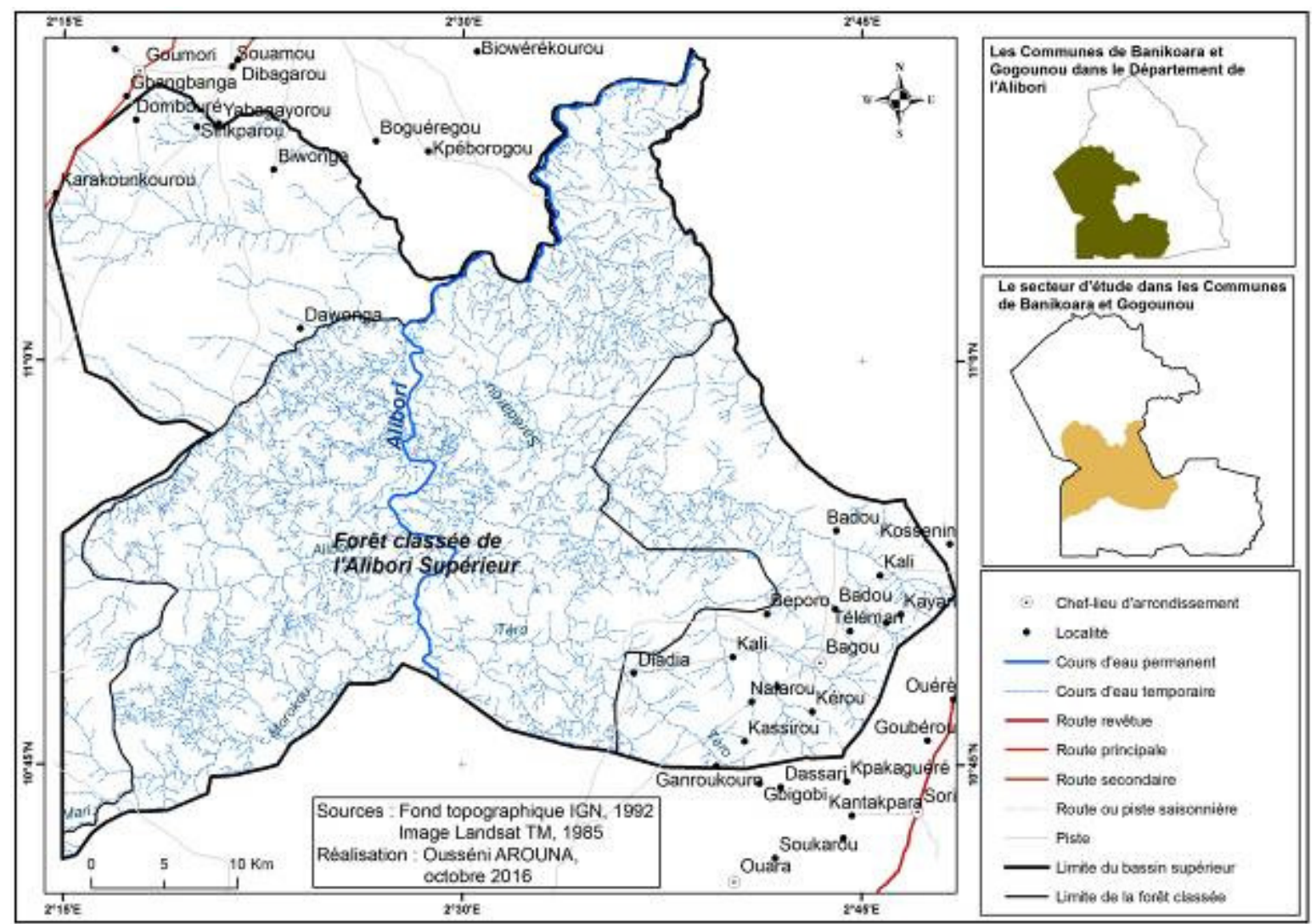

Figure 1 : situation géographique du bassin supérieur de la rivière Alibori

Le bassin supérieur de la rivière Alibori se trouve dans une région à climat soudanien. La végétation climacique devrait être constituée de forêts denses sèches et de forêts claires (White, 1983). Mais sous l'effet, de l'agriculture, de l'élevage et de l'exploitation forestière on y observe des savanes arbustives et des mosaïques de champs et jachères et quelques îlots de forêts claires et de savanes boisées.

Matériel de recherche : Les scènes d'images Landsat 5 TM (P192/R052 et P192/R053) du 31 juillet 1985 et d'images Landsat 8 OLI-TIRS (P192/R052 et P192/R053) du 28 août 2016 de $30 \mathrm{~m}$ de résolution spatiale, téléchargées sur le site de earthexplorer.usgs.gov ont été utilisées.

Méthodes de cartographie diachronique de l'occupation des terres : Le mosaïquage des quatre (4) scènes d'images deux à deux avec le logiciel Erdas Imagine 14.00 et des améliorations radiométriques pour rehausser la lisibilité des images à l'aide du logiciel Envi 5.1 ont d'abord été effectués. L'interprétation numérique des images Landsat 5 TM de 1985 et des images Landsat 8 OLI-TIRS a été réalisée selon la méthode de classification supervisée à l'aide du logiciel Envi 5.1. Les aires d'entrainement ont été délimitées au pixel près sur l'ensemble des unités d'occupation des terres. Le maximum de vraisemblance est l'algorithme de classification appliqué.

Contrôle-terrain : Pour le contrôle-terrain,-80 points GPS ont été relevés dans les différentes unités d'occupation des terres afin de confronter les classes issues de l'interprétation numérique des images et des données collectées sur le terrain. A partir des données GPS et de celles issues de l'interprétation des images, une matrice de confusion a été établie pour calculer la précision globale de cette interprétation numérique des images en vue de sa validation.

Restitution cartographique: La restitution cartographique a été réalisée à l'aide du logiciel ArcGIS 10.3 en respectant les règles de la sémiologie graphique. Qualitativement, les mêmes classes d'occupation des terres ont été observées au cours des deux années (1985 et 2016).

Matrice de transition : La matrice de transition est un tableau constitué de $X$ lignes et de $Y$ colonnes. Le nombre $x$ de lignes de la matrice indique le nombre d'unités d'occupation des terres en 1985 et le nombre $Y$ 

végétation dans le bassin supérieur de l'Alibori au Benin

de colonnes de la matrice correspond aux unités d'occupation des terres de 2016. Les transformations se font donc des lignes vers les colonnes. La diagonale de la matrice est constituée de superficies des unités d'occupation des terres restées inchangées entre 1985 et 2016. Les superficies des unités d'occupation des terres ont été calculées à partir du croisement des couches d'occupation des terres de 1985 et 2016 à l'aide de la fonction Intersect de l'interface Arctoolbox du logiciel ArcGIS 10.3.

Méthode d'évaluation de l'état de la biodiversité végétale

Relevés phytosociologiques et inventaire forestier: Les relevés phytosociologiques et l'inventaire forestier ont permis de caractériser l'état de la biodiversité végétale dans le bassin supérieur de l'Alibori. Les relevés phytosociologiques ont été effectués suivant la méthode sigmatiste de Braun-Blanquet (1932). Cette méthode est basée sur le principe d'homogénéité floristique et d'uniformité topographique. Elle a été déjà utilisée par plusieurs auteurs (Toko Imorou, 2008 ; Arouna, 2012). L'aire de relevé retenue est de $100 \mathrm{~m}^{2}$ pour la strate herbacée et de $900 \mathrm{~m}^{2}$ pour la strate ligneuse. Au total 150 placeaux ont été installés à raison de 115 placeaux dans la forêt classée et 35 placeaux dans les terroirs villageois. Des coefficients d'abondance-dominance qui sont relatifs à l'espace occupé par l'ensemble des individus de chaque espèce ont été utilisés en se référant aux travaux Toko Imorou (2008) et de Arouna (2012).

Traitement des données phytosociologiques

Paramètres de diversité spécifique

Richesse spécifique (R) : Elle correspond au nombre d'espèces que compte une communauté ou un peuplement (Ramade, 1994).

Indice de diversité de Shannon (H)

\section{RESULTATS}

Dynamique de l'occupation des terres de 1985 à 2016 : Les précisions globales des cartes d'occupation des terres de 1985 et de 2016 sont respectivement de 92 et de $95 \%$.

Physionomie du bassin supérieur en 1985 et $2016: \mathrm{La}$ physionomie du bassin supérieur de la rivière Alibori en 1985 et en 2016 est présentée par les figures 2 et 3. L'examen de la figure 2 révèle que le bassin supérieur de la rivière Alibori en 1985 était dominé par les formations végétales naturelles notamment les forêts galeries, les forêts denses sèches, les forêts claires et les savanes

$$
\mathbf{H}=-\Sigma \text { Pilog }{ }_{2} \mathbf{P i}
$$

Avec $\mathrm{Pi}=(\mathrm{ni} / \mathrm{N})$ est la fréquence relative des individus de l'espèce (i) ; (ni) est le nombre d'individu (s) de l'espèce (i) ; (N) est le nombre total d'individus recensés. Cet indice varie généralement de 0 à 5 bits. Les valeurs élevées de $\mathrm{H}$ traduisent les conditions favorables du milieu pour l'installation de nombreuses espèces (Dajoz, 1985).

Equitabilité de Pielou: L'équitabilité de Pielou est obtenue à partir de la formule suivante :

$$
E=\frac{H}{\log _{2} R}
$$

E varie de 0 à 1. L'équitabilité de Piélou élevé est le signe d'un peuplement équilibré (Dajoz, 1985). Par contre les valeurs faibles correspondent à la présence d'un nombre élevé d'espèces rares ou d'un petit nombre d'espèces dominantes.

Spectres biologiques et phytogéographiques

Spectres biologiques : Les spectres biologiques sont calculés à partir des types biologiques. L'identification des types biologiques des espèces a été réalisée sur la base des travaux de Raunkiaer (1934). Ainsi, cinq types biologiques ont été recensés : les phanérophytes, les thérophytes, les chaméphytes, les géophytes et les hémicryptophytes.

Spectres phytogéographiques: Les spectres phytogéographiques sont calculés à partir des types phytogéographiques. Les types phytogéographiques des espèces ont été établis à partir des subdivisions chorologiques de White (1983). Ainsi, on a distingué les espèces à large distribution géographique, les espèces à distribution continentale et les espèces de l'élément-base (espèces soudaniennes).

boisées. Dans les terroirs villageois, on observait majoritairement des mosaïques de champs et de jachères. En 2016 la physionomie de ce territoire a totalement changé surtout dans la forêt classée (Figure 3). En effet, dans la forêt classée on observe majoritairement des mosaïques de champs et de jachères et des savanes arborées et arbustives. II n'y a pratiquement plus de différence en termes de physionomie entre la forêt classée et les terroirs villageois. La forêt classée de l'Alibori supérieur est pratiquement devenue une véritable zone agricole. 


\section{Arouna et al., J. Appl. Biosci. 2016 Dynamique de l'occupation des terres et état de la flore et de la}

végétation dans le bassin supérieur de l'Alibori au Benin

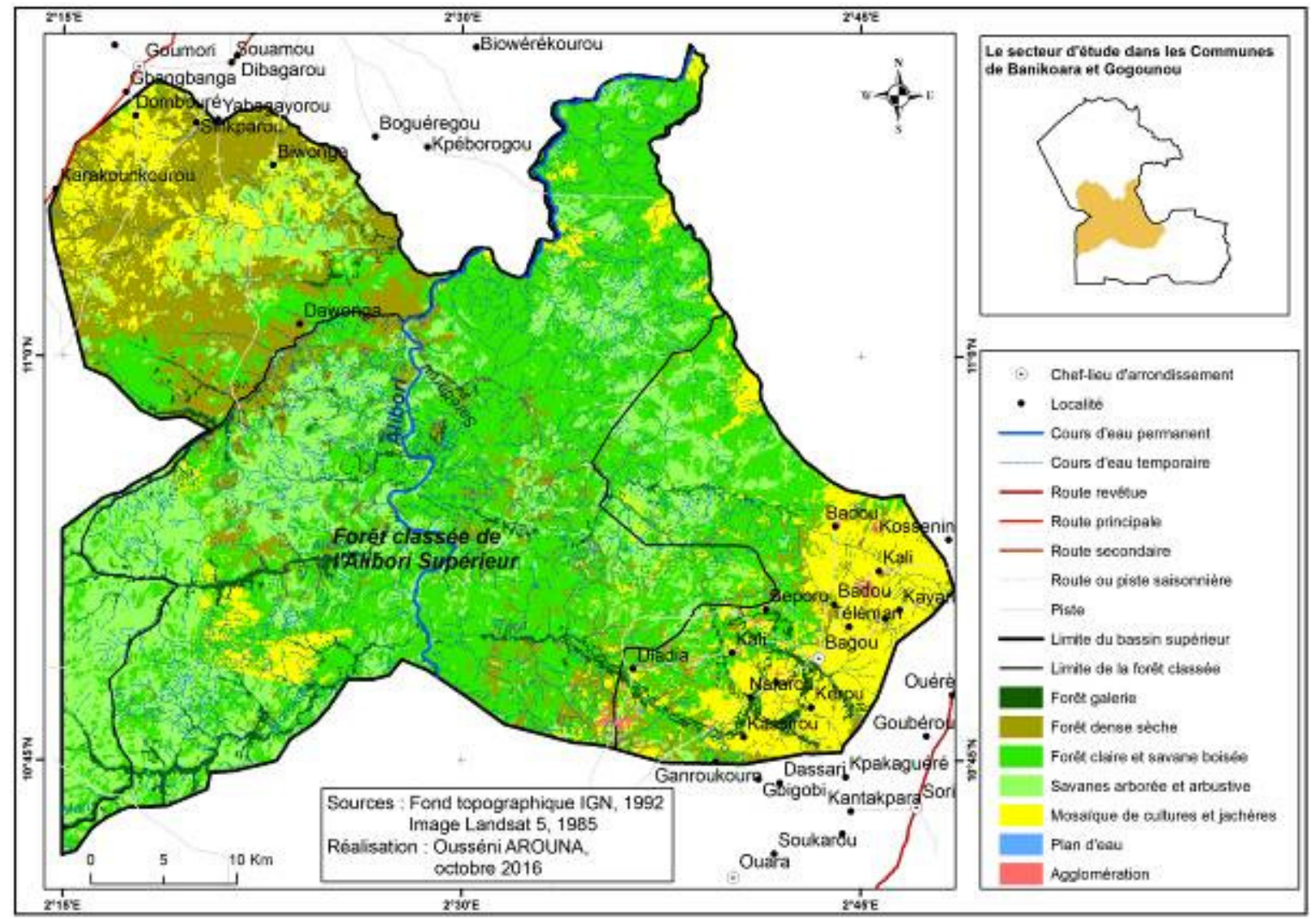

Figure 2 : Occupation des terres dans le bassin supérieur de l'Alibori en 1985 
Arouna et al., J. Appl. Biosci. 2016 Dynamique de l'occupation des terres et état de la flore et de la végétation dans le bassin supérieur de l'Alibori au Benin

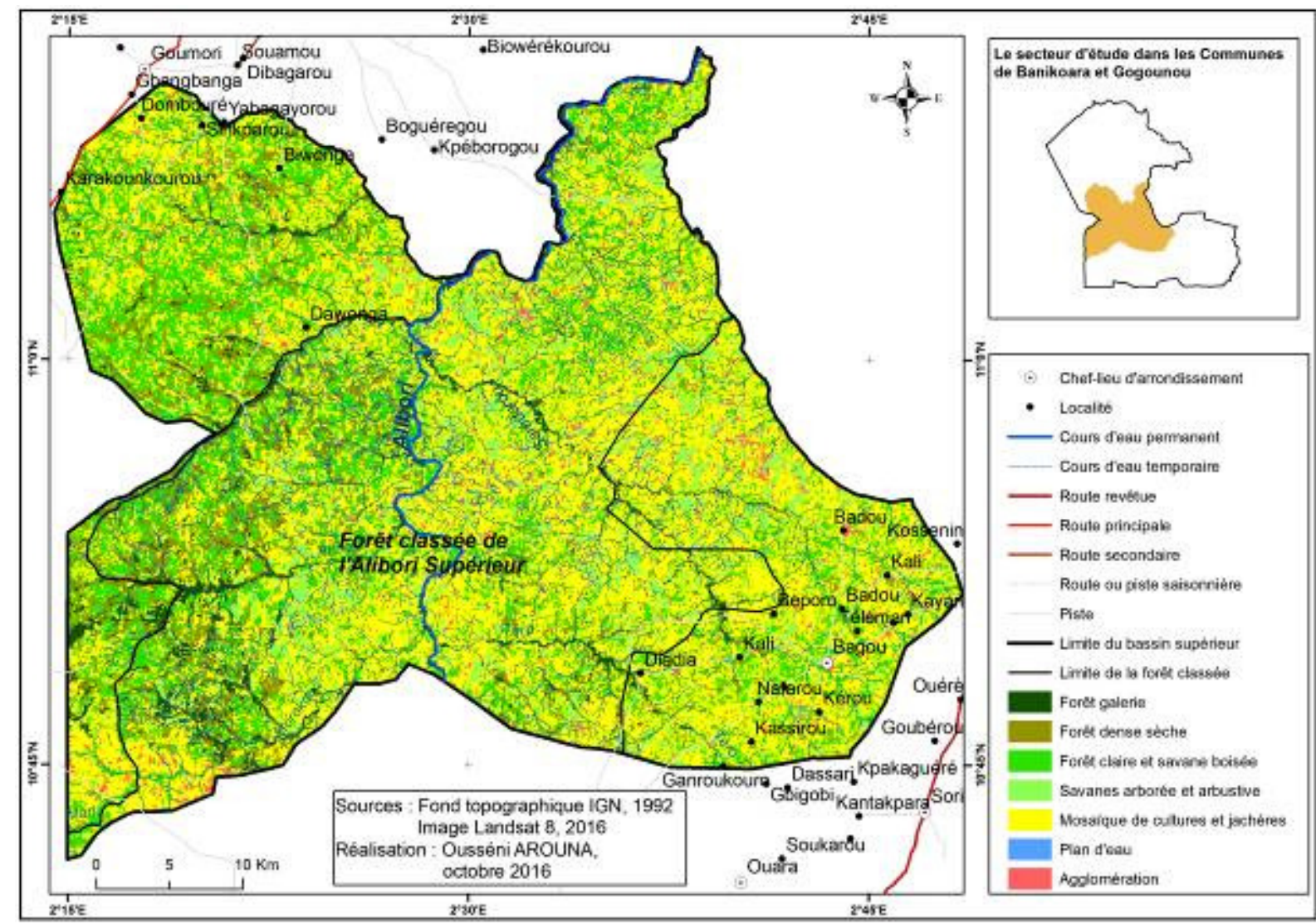

Figure 3 : occupation des terres dans le bassin supérieur de l'Alibori en 2016

Détection de changements au niveau des formations végétales de 1985 et 2016 : Les changements observés au niveau des formations végétales en1985 et en 2016 sont exprimés par les différentes formes de conversion analysées à partir de la matrice de transition (Tableau 1).

Tableau 1 : Matrice de transition de l'occupation des terres $\left(\mathrm{Km}^{2}\right)$ entre 1985 et 2016 dans le bassin supérieur de l'Alibori

\begin{tabular}{|l|c|c|c|c|c|c|c|c|}
\hline \multicolumn{10}{|c|}{$\mathbf{2 0 1 6}$} \\
\hline $\mathbf{1 9 8 5}$ & FDS & FG & FCSB & SAA & MCJ & PE & AG & Total \\
\hline FDS & 61,00 & 0,00 & 98,39 & 19,78 & 130,58 & 0,00 & 5,94 & 315,68 \\
\hline FG & 0,00 & 48,00 & 0,00 & 0,00 & 23,31 & 0,00 & 0,20 & 71,51 \\
\hline FCSB & 53,49 & 0,00 & 165,00 & 73,46 & 365,73 & 0,00 & 7,85 & 665,53 \\
\hline SAA & 95,30 & 0,00 & 163,70 & 107,00 & 437,57 & 0,00 & 5,25 & 808,82 \\
\hline MCJ & 0,00 & 0,49 & 0,00 & 4,51 & 144,00 & 0,15 & 5,59 & 154,75 \\
\hline PE & 0,00 & 0,10 & 0,00 & 0,10 & 0,29 & 1,00 & 0,00 & 1,49 \\
\hline AG & 0,29 & 0,00 & 0,00 & 0,15 & 0,00 & 0,00 & 8,00 & 8,44 \\
\hline Total & 210,08 & 48,59 & 427,09 & 205,00 & 1101,48 & 1,15 & 32,83 & 2026,22 \\
\hline
\end{tabular}

FDS : forêt dense ; FG : forêt galerie ; FCSB : forêt claire et savane boisée ; SAA : savanes arborée et arbustive ; MCJ : Mosaïque de champs et jachères ; $P E$ : plan d'eau ; $A G$ : Agglomération

L'examen de la matrice de transition permet de retenir que les 7 classes d'occupation des terres observées en 1985 sont maintenues en 2016. Cependant, il est important de souligner que les superficies de ces différentes classes d'occupation des terres ont connu d'importants changements. Globalement, il est remarqué 
une forte conversion des classes naturelles vers les classes anthropiques, des classes naturelles entre elles et accessoirement des conversions des classes anthropiques vers les classes naturelles. Des formes de stabilité ont été aussi observées.

\section{Etat de la flore et de la végétation}

Composition floristique et diversité spécifique : Le recensement floristique dans les différentes formations végétales du bassin supérieur de la rivière Alibori a permis de dénombrer 183 espèces dont $49 \%$ de ligneux et $51 \%$ d'herbacées. Les familles les plus représentées sont les poaceae. Le tableau II fait la synthèse des différents paramètres de diversité spécifique des formations végétales.

Tableau 2 : Paramètres de diversité spécifique par formation végétale

\begin{tabular}{lccc}
\hline Formations végétales & $\mathrm{R}$ (Nombre d'espèces/Placeau) & $\mathrm{H}(\mathrm{Bit})$ & $\mathrm{E}$ \\
\hline Forêts galeries & $18 \pm 4$ & $1,51 \pm 0,43$ & $0,87 \pm 0,05$ \\
Forêts claires et savanes boisées & $24 \pm 5$ & $2,56 \pm 0,40$ & $0,82 \pm 0,08$ \\
Savanes arborées et arbustives & $30 \pm 6$ & $2,69 \pm 0,72$ & $0,80 \pm 0,12$ \\
\hline
\end{tabular}

$\mathrm{R}$ : Richesse spécifique ; $\mathrm{H}$ : Indice de diversité de Shannon ; $\mathrm{E}$ : Equitabilité de Pielou

L'examen du tableau 2 révèle que les savanes arborées et arbustives sont les plus diversifiées suivies de très près par les forêts claires et savanes boisées. Les valeurs élevées des paramètres de diversité spécifique dans les forêts claires et les différents types de savanes constituent un espoir pour reconstituer les formations végétales dégradées.

Types biologiques et phytogéographiques des formations naturelles : Les figures 4 et 5 présentent les types biologiques et phytogéographiques des formations naturelles.

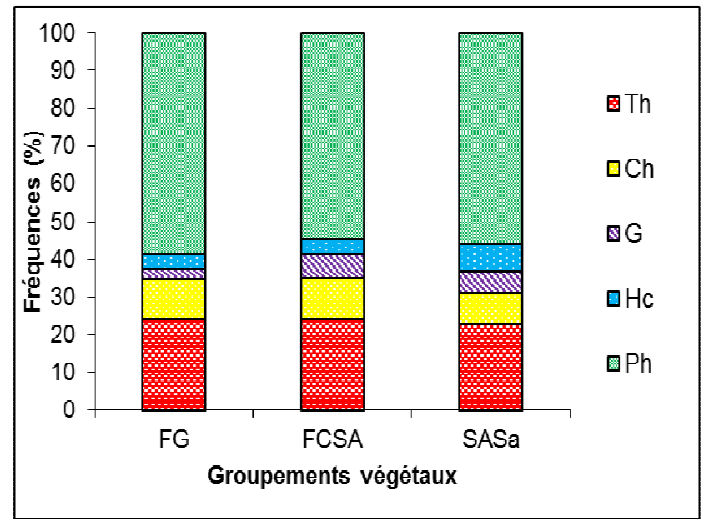

(a) : Spectres bruts

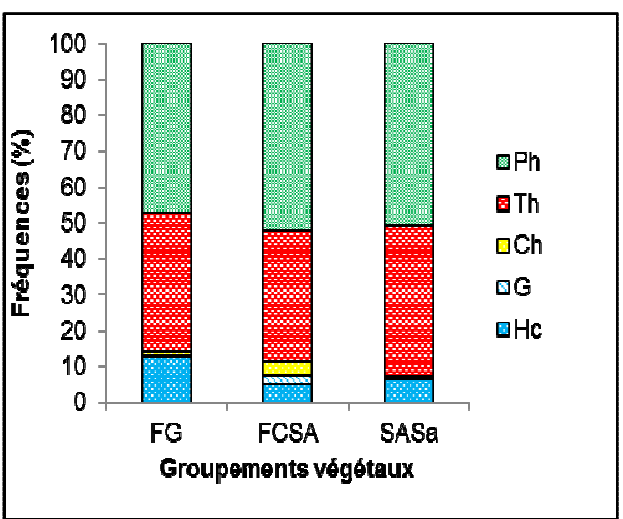

(b) : Spectres pondérés

Figure 4 : Spectres bruts et pondérés des types biologiques des formations naturelles

Légende : Th : thérophytes; $\mathrm{Ch}$ : chaméphytes ; G : géophytes ; $\mathrm{Hc}$ : hémicryptophytes ; Ph : phanérophytes ; $F G$ : forêts galeries ; FCSA : forêts claires et savanes boisées ; SASa : savanes arborées et savanes arbustives.

L'examen de la figure 4 révèle que les phanérophytes sont les formes de vie les plus abondantes et dominantes suivies par les thérophytes. La présence importante des thérophytes est un indicateur de dégradation des formations végétales. 


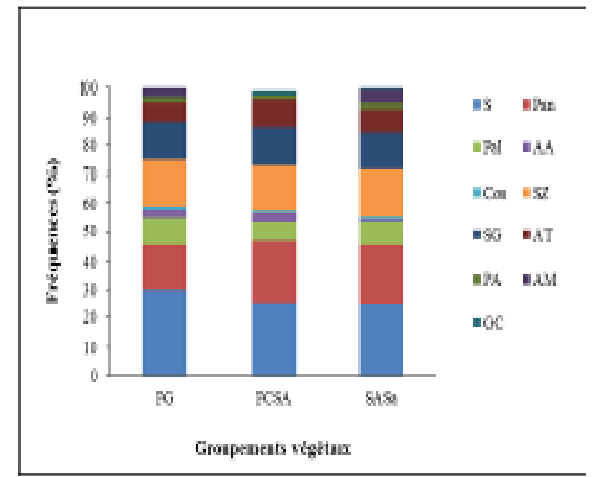

(a) : Spectres bruts

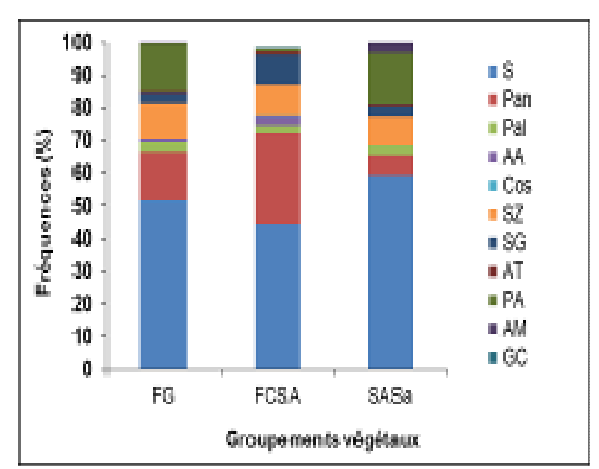

(b) : Spectres pondérés

Figure 5 : Spectres bruts et pondérés des types phytogéographiques des formations naturelles

Légende : $S$ : espèces soudaniennes ; Pan : espèces pantropicales ; Pal : espèces paléotropicales ; $\mathrm{AA}$ : espèces afro-américaines ; Cos : espèces cosmopolites ; $S Z$ : espèces soudano-zambéziennes ; $S G$ : espèces soudano-guinéennes ; AT : espèces réparties dans toute l'Afrique tropicale ; PA : espèces réparties dans plusieurs régions d'Afrique; AM : espèces réparties en Afrique et à Madagascar ; GC : espèces guinéo-congolaises ; FG : forêts galeries ; FCSA : forêts claires et savanes boisées ; SASa : savanes arborées et savanes arbustives.

L'examen de la figure 5 montre l'abondance et la dominance des espèces soudaniennes. La présence remarquable des espèces pantropicales qui sont des

\section{DISCUSSION}

Facteurs sous-jacents de la dynamique de l'occupation des terres : L'évaluation de la dynamique de l'occupation des terres dans le bassin supérieur de l'Alibori de 1985 à 2016 a révélé une régression des formations naturelles au profit des mosaïques de champs et jachères et des agglomérations même à l'intérieur de la forêt classée de l'Alibori Supérieur. II se produit dans cette forêt classée la déforestation et la dégradation des forêts qui méritent une attention particulière. La pratique des activités comme l'agriculture, l'élevage, l'exploitation forestière a été indexée par plusieurs auteurs (Arouna 2002 ; Mama, 2013 ; Issiako, 2015) pour expliquer la dégradation des formations naturelles dans la forêt classée de l'Alibori Supérieur. Au-delà de considérer ces activités comme les moteurs de la déforestation et de la dégradation du couvert végétal, il est opportun de se demander les fondements qui peuvent expliquer la pratique de ces activités dans la forêt classée de l'Alibori Supérieur dotée pourtant d'un plan d'aménagement qualifié de participatif. Le bassin supérieur de la rivière Alibori a été érigé en forêt classée par l'administration coloniale à travers l'arrêté №6459 SE du 18 août 1955 (Arouna, 2002). A cette époque, les populations riveraines ne semblent pas être associées à cette opération (Arouna, 2002). Avec l'explosion démographique et ses corollaires d'augmentation de besoins en terres cultivables couplée à l'interdiction espèces à large distribution géographique dans les formations végétales montre bien l'état de perturbation de ces formations.

d'accès aux forêts classées sans les moyens de la faire respecter, la voie de libre accès aux terres de cette forêt classée a été ouverte. L'appropriation de l'ensemble des forêts classées par l'Etat et l'exclusion des populations riveraines, souvent faites au nom de l'intérêt général, ont légitimé les pratiques dévastatrices au sein de ces forêts classées. L'Etat, à travers l'administration forestière, n'exerce plus qu'un contrôle superficiel sans activités d'aménagement et de surveillance dans la forêt classée de l'Alibori Supérieur (Arouna, 2002 ; Djogbénou et al., 2011). La forêt classée de l'Alibori Supérieur est alors devenue le théâtre de plusieurs activités anthropiques: agriculture, élevage, exploitation forestière, braconnage, feux de végétation. II s'est alors progressivement instauré la «tragédie des biens communs» (Hardin, 1968), symbolisant la dégradation de l'environnement suite à l'utilisation concurrente d'une ressource par plusieurs usagers, chacun cherchant à tirer le maximum de profit. Implications de la dynamique de l'occupation des terres sur la flore et la végétation : La caractérisation de la flore et de la végétation a révélé que les valeurs des paramètres de diversité spécifique sont plus faibles dans les forêts galeries. Une faible valeur d'un indice de diversité spécifique notamment l'indice de Shannon est le signe d'un milieu très peu stable (Dajoz, 1985). Les forêts galeries du bassin supérieur de l'Alibori sont alors perturbées. Les formes de vie les plus abondantes sont 
les phanérophytes fortement concurrencées par les thérophytes dans la plupart des formations végétales. L'abondance et la dominance des phanérophytes montrent la forte représentativité des ligneux dans presque toutes les formations végétales. Cette forte présence des phanérophytes constitue un espoir pour reconstituer les formations naturelles dégradées. Toutefois, la présence remarquable des thérophytes dans les forêts claires, les savanes boisées, les savanes arborées et arbustives est le signe évident de perturbation de ces formations naturelles (Arouna 2012, Gbesso et al., 2013). Les types phytogéographiques ont été aussi évalués à travers leurs spectres bruts et pondérés. Les types phytogéographiques traduisent la fidélité des espèces à leur région de confinement et permettent de juger de la spécificité de la flore locale (Arouna, 2012). Dans l'ensemble, les espèces soudaniennes sont les plus abondantes et les plus dominantes attestant que la flore de la région soudanienne garde encore sa spécificité

\section{CONCLUSION}

La cartographie diachronique de l'occupation des terres et les inventaires phytosociologique et forestier ont révélé que les formations forestières naturelles ont régressé au profit des mosaïques de champs et jachères avec une perturbation de la flore et de la végétation dans le bassin supérieur de la rivière Alibori. Les conversions des formations naturelles vers les formations anthropiques sont les changements les plus observés. Les valeurs des paramètres de diversité spécifique sont relativement faibles attestant d'une dégradation de ces formations végétales. L'abondance et la dominance des phanérophytes sont concurrencées par celles des thérophytes. Cette présence remarquable des thérophytes est un indicateur qui confirme la perturbation des formations végétales déjà révélées par les images

\section{REFERENCES BIBLIOGRAPHIQUES}

Arouna O, 2012. Cartographie et modélisation prédictive des changements spatio-temporels de la végétation dans la commune de Djidja au Bénin : implications pour l'aménagement du territoire. Thèse de Doctorat Unique, Université d'Abomey-Calavi, Bénin, $246 \mathrm{p}$.

Arouna 0, 2002. L'exploitation des ressources biologiques et la dynamique de la forêt classée de l'Alibori supérieur (secteur de l'arrondissement de Bagou), Mémoire de maîtrise, UAC/FLASH/DGAT, $115 \mathrm{p}$. phytogéographique en dépit des perturbations enregistrées. Toutefois, l'importance remarquable des espèces pantropicales dans la plupart des formations végétales naturelles hormis la forêt galerie atteste bien l'existence des perturbations anthropiques qui peuvent bouleverser la répartition spatiale des plantes à moyen et long termes. L'abondance et la dominance des espèces soudaniennes dans les forêts galeries méritent qu'on y accorde une attention particulière. En principe, les forêts galeries constituent les refuges des espèces forestières en régions soudanienne et soudano-guinéenne. L'abondance et la dominance des espèces soudaniennes dans les forêts galeries du bassin supérieur de la rivière Alibori est un indicateur évident de la perturbation de la phytogéographie. Au total, l'hypothèse de la présente recherche qui stipule que la dynamique régressive de l'occupation des terres induit une baisse de la diversité spécifique et une perturbation de la répartition des espèces végétales est alors vérifiée.

satellites et les paramètres de diversité spécifique. L'examen des types phytogéographiques a révélé que les espèces soudaniennes sont les plus abondantes et les plus dominantes attestant que la flore du milieu d'étude garde encore sa spécificité phytogéographique en dépit des perturbations enregistrées. Toutefois, l'importance remarquable des espèces pantropicales dans la plupart des formations végétales naturelles hormis la forêt galerie atteste bien de l'existence de fortes pressions anthropiques qui peuvent perturber la phytogéographie du milieu à moyen et à long termes. L'ensemble de ces perturbations peut avoir des impacts négatifs sur la biodiversité si des mesures adéquates d'aménagement intégré du territoire ne sont pas prises très tôt.

Braun-Blanquet J, 1932. Plant sociology: The study of plant communities. English translated revised and edited by Fuller G. D. \& Conard H. S, 439 p.

Dajoz R, 1985. Précis d'écologie. Bordas, Paris, France, $504 \mathrm{p}$.

Djogbénou C. P., Arouna O, Toko Imorou I \& Sinsin B, 2011. Analyse comparative des profils des plans d'aménagement participatifs des forêts classées au Bénin. Rev. Sc. Env. Uni. Lomé (Togo), $7: 51-79$

Gbesso G H F, Lougbegnon T O, Tente A H B, Mensah H A G \& Sinsin A B, 2013. Caractérisations 
phytoécologique et structurale des groupements végétaux abritant Chrysophyllum albidum (G. Don) sur le plateau d'Allada au Sud-Benin, Afrique Science 09 (3) (2013), 147-158.

Hardin G, 1968. The Tragedy of Commons. Science, 162: 1243-1248.

Issiako, D, 2015. Dynamique de l'occupation du sol dans le bassin supérieur de la rivière Alibori (secteur Banikoara-Gogounou). Mémoire de maîtrise, Département de Géographie et Aménagement du Territoire, Université d'Abomey-Calavi, Bénin, $59 \mathrm{p}$.

Mama A, Sinsin B., De Cannière C. \&. Bogaert J., 2013. Anthropisation et dynamique des paysages en zone soudanienne au nord du Bénin. TROPICULTURA, 31 (1) : 78-88

Ramade F, 1994.- Éléments d'écologie. Écologie fondamentale 2. Ediscience international, Paris, $579 \mathrm{p}$.

Raunkiaer, C, 1934. The life forms of plants and statistical plant geography. Clarendron Press, Oxford, $632 \mathrm{p}$.

Sabi Monra A, 2015. Gestion des terroirs et conservation de la biodiversité végétale dans l'Arrondissement de Bagou (Commune de Gogounou). Mémoire de maîtrise, Département de Géographie et Aménagement du Territoire, Université d'Abomey-Calavi, Bénin, $82 \mathrm{p}$.

Sow 0,2012 . Caractéristiques de la végétation ligneuse et impact des traitements sylvicole dans la dynamique évolutive des forêts naturelles : cas de la forêt classée du Nazinou dans le centreOuest du Burkina Faso. Mémoire, Institut du développement rural/Burkina Faso.

Toko Imorou I, Arouna O \& Sinsin B, 2010. Cartographie des changements spatio-temporels de l'occupation du sol de la forêt classée de l'Alibori Supérieur au Nord-Bénin. BenGéo., 7 : 22-39

Toko Imorou I, 2008. Etude de la variabilité spatiale de la biomasse herbacée, de la phénologie et de la structure de la végétation le long des toposséquences du bassin supérieur du fleuve Ouémé au Bénin. Thèse de Doctorat Unique, Université d'Abomey-Calavi, $241 \mathrm{p}$.

White $F, 1983$. The vegetation of Africa. A descriptive memoir to accompany the UNESCO/ AETFAT/UNSO vegetation map of Africa. UNESCO, Paris, France, $356 \mathrm{p}$. 